\title{
A single nucleotide polymorphism in EZH2 predicts overall survival rate in patients with cholangiocarcinoma
}

\author{
ELISA PAOLICCHI ${ }^{1}$, PAOLA PACETTI ${ }^{2}$, ELISA GIOVANNETTI ${ }^{3}$, ANDREA MAMBRINI ${ }^{2}$, MASSIMO ORLANDI $^{2}$, \\ FRANCESCO CREA $^{1}$, ANTONELLO A. ROMANI ${ }^{4}$, ROBERTA TARTARINI ${ }^{2}$, ROMANO DANESI ${ }^{1}$, \\ GODEFRIDUS J. PETERS ${ }^{3}$ and MAURIZIO CANTORE ${ }^{2}$ \\ ${ }^{1}$ Department of Internal Medicine, Division of Pharmacology, University of Pisa, Pisa I-56126; ${ }^{2}$ Department of Oncology, \\ Carrara Civic Hospital, Piazza Sacco e Vanzetti, Carrara I-54033, Italy; ${ }^{3}$ Department of Medical Oncology, \\ VU University Medical Center, Amsterdam, Amsterdam 1081HV, The Netherlands; ${ }^{4}$ Department of Surgical Sciences, \\ Division of Surgical Clinic and Organ Transplant, University of Parma, Parma I-43100, Italy
}

Received May 23, 2012; Accepted August 6, 2012

DOI: $10.3892 / \mathrm{ol} .2013 .1559$

\begin{abstract}
Cholangiocarcinoma (CCA) is a deadly disease arising from the malignant transformation of cholangiocytes. Enhancer of zeste homolog 2 (EZH2) is overexpressed in poorly differentiated CCA. Functional single nucleotide polymorphisms (SNPs) in this gene may affect the role of EZH2 in cholangiocarcinogenesis and chemoresistance. The aim of the current study was to evaluate the correlation between EZH2 SNPs and clinical outcome. Using PROMO3.0, GeneCard and MicroSNiper, 4 EZH2 SNPs with functional relevance in CCA were selected in silico. These SNPs were studied in genomic DNA extracted from the blood samples of 75 patients with advanced CCA, who were treated with epirubicin-cisplatin-xeloda (ECX regimen). SNP genotyping was performed with specific PCR assays. The rs887569 TT genotype was correlated with a significantly longer overall survival (OS; TT vs. CT-CC, $\mathrm{P}=0.026$ ). Moreover, the TT genotype revealed a trend toward a significant association with a reduced risk of mortality (HR, 0.59; 95\% CI, 0.33-1.05; $\mathrm{P}=0.075$ ), by multivariate analysis. These results support future studies on the role of rs887569 EZH2 SNP as a possible predictive marker of OS in advanced CCA patients.
\end{abstract}

\section{Introduction}

Cholangiocarcinoma (CCA) is a malignancy arising from the biliary tract epithelium (1). CCA is rare, with an annual incidence of 2-3 cases per 100,000 individuals in the Western population (2). However, it is the second most common primary hepatic malignancy, with recent epidemiological studies

Correspondence to: Dr Elisa Paolicchi, Department of Internal Medicine, Division of Pharmacology, University of Pisa, Via Roma 67, Pisa, I-56126, Italy

E-mail: eli.paolicchi@gmail.com

Key words: polycomb, EZH2, cholangiocarcinoma, ECX regimen, polymorphism suggesting a progressively increasing incidence in Western countries (3-5). Anatomically, CCA is classified as intrahepatic (IH-CCA) or extrahepatic (EH-CCA). IH-CCA arises from the intrahepatic ducts, which extend from the periphery of the liver to the second-order bile ducts within the liver (6). EH-CCA is observed and defined in three different growth patterns: periductal infiltrating, papillary or intraductal and mass forming (7). The high mortality rate of CCA is mainly due to its aggressive behavior. Indeed, the majority of these tumors are diagnosed at a late stage of disease progression, precluding surgical therapies $(8,9)$. Furthermore, CCA is characterized by a marked resistance to chemotherapy $(10,11)$. Several drugs have been tested in phase II studies in unresectable CCA (5-fluorouracil, gemcitabine methasulfon-m-anisidide, cisplatin, rifampicin, mitomycin $\mathrm{C}$ and paclitaxel) with partial response rates below $9 \%$ and average survival shorter than 12 months (8). Therefore, studies for the identification of key factors that play a critical role in tumor chemosensitivity/resistance for the selection of patients with the highest likelihood of responding to these therapies are urgently required.

Polycomb group $(\mathrm{PcG})$ proteins are epigenetic chromatin modifiers involved in cancer development and their roles are now being evaluated in numerous human malignancies (12). PcG proteins are essential for cancer stem cell (CSC) selfrenewal. PcG members are organized in two main protein complexes: Polycomb repressive complex 1 and 2 (PRC1, PRC2) (13). PRC2 is required in the initial stage of silencing, through the histone H3 Lys 27 trimethylation (14) which contributes to the recruitment of PRC1. PRC1 is required for stable maintenance of the initiated PcG gene silencing, through histone $\mathrm{H} 2 \mathrm{~A}$ ubiquitylation (14), on specific target loci. Enhancer of zeste homolog $2(\mathrm{EZH} 2)$ is the catalytic subunit of PRC2; while B-cell-specific Moloney murine leukemia virus integration site 1 (BMI-1) contributes to the ubiquitin E3 ligase activity in PRC1. EZH2 is overexpressed in poorly differentiated CCA (15). Several genetic and epigenetic factors may be involved in the deregulation and modulation of key signaling pathways in tumor aggressiveness and chemoresistance. The abnormal expression of EZH2 is involved in the tumorigenic 
processes and is regarded as a potential marker of aggressive types of cancer with poor prognoses $(16,17)$. Previous studies have demonstrated that EZH2 contributes to the epigenetic silencing of several target genes that control cell growth and proliferation, including E-cadherin, Rb and p16 (18). The overexpression of EZH2 may induce hypermethylation of the promoter of the p16 gene, reducing the expression of p16, which is a key step in the multistep cholangiocarcinogenesis from hepatolithiasis to intraepithelial neoplasia (19).

A recent study described 26 single nucleotide polymorphisms (SNPs) in the EZH2 locus, including SNPs correlated with lung cancer risk (20). Genetic analysis of germinal variants may be an easy-to-perform prognostic tool, readily transferable into the clinic. Therefore, the study of candidate polymorphisms of EZH2 as biomarkers of clinical outcome may provide effective prognostic markers in CCA patients. The aim of the current study was to evaluate a correlation between EZH2 SNPs and clinical outcome in CCA patients.

\section{Materials and methods}

Patients. A total of 75 patients with histologically confirmed unresectable biliary tract cancer were enrolled in this retrospective pharmacogenetic single-center study at the Department of Oncology of Carrara Civic Hospital (Carrara, Italy), between February 2004 and November 2010. CCA patients were treated upfront with intravenous or intra-arterial cisplatin (Platinol ${ }^{\circledR}$, Bristol-Myers Squibb, Roma, Italy) and epirubicin (Pharmorubicin ${ }^{\circledR}$, Pfizer Italia S.R.L., Latina, Italy) and oral capecitabine $\left(\right.$ Xeloda $^{\circledR}$, Roche S.p.A, Milano, Italy; ECX regimen). This study was approved by the ethics review board of Carrara Hospital. Informed consent was obtained from all the patients.

DNA isolation. Genomic DNA was extracted from peripheral venous blood samples $(5 \mathrm{ml})$ from an antecubital vein of $75 \mathrm{CCA}$ patients and stored anonymously at $-20^{\circ} \mathrm{C}$ in the Laboratory of VU University Medical Center, Department of Medical Oncology (Amsterdam, The Netherlands). Genomic DNA was isolated through the QIAamp DNA mini kit (Qiagen, Venlo, The Netherlands). The purity and quantity of DNA obtained were measured by spectrophotometer NanoDrop ${ }^{\circledR}$-1000-Detector (NanoDrop-Technologies, Wilmington, DE, USA). The absorbance was read at 260 and $280 \mathrm{~nm}$ and the contamination by proteins was estimated through the calculation of 260/280 ratio.

In silico analysis. A total of 26 previously described EZH2 SNPs were functionally tested by the appropriate software. SNPs were screened through in silico characterization based on functional relevance [missense mutation, transcription factor binding (TFB), miRNA binding]. In particular, the PROMO3.0 (http://alggen.lsi.upc.es/cgibin/ promo_v3/promo/promoinit.cgi?dirDB $=$ TF_8.3) in which human factors and binding sites were considered, with a maximum matrix dissimilarity rate of $15(21,22)$, GeneCard (http://www.genecards.org/) and MicroSNiper (http://cbdb. nimh.nih.gov/microsniper) software were used (23). g.148519011C >T (rs6464926), g.148517456T $>$ G $($ rs17171119)
Table I. Patient characteristics.

Characteristics

No. of patients

\begin{tabular}{lc}
\hline General & \\
Total & 75 \\
Evaluable disease & 74 \\
Median age (range), years & $62.3(26-80)$ \\
Gender & \\
Male & 44 \\
Female & 31 \\
ECOG & \\
0 & 35 \\
1 & 30 \\
2 & 10
\end{tabular}

Tumor diagnosis

Intrahepatic cholangiocarcinoma $\quad 56$

Gallbaldder carcinoma $\quad 11$

Common bile duct 8

Sites of metastases

Liver 60

Involvement $<50 \% \quad 40$

Involvement $>50 \% \quad 20$

Lymph nodes $\quad 10$

Peritoneum 7

Local disease recurrence $\quad 6$

Other 4

Median Ca19.9 level (range, IU/ml) 204 (0-11400)

Involvement represents the percentage of liver tissue with metastatic cells.

and g.148505302 C>T (rs887569) were analyzed with real-time PCR. Applied Biosystems SNP genotyping assays were used for reactions. The PCR assays were performed using $20 \mathrm{ng}$ of genomic DNA diluted in $5.94 \mu \mathrm{l}$ DNase RNase free water, $6.25 \mu \mathrm{l}$ of TaqMan Universal Master Mix (Applied Biosystems, Foster City, CA, USA) with AmpliTaq Gold and $0.3125 \mu \mathrm{l}$ of the assay mix (specific primers and probe) in $12.5 \mu$ l total volume. The allelic content of each sample in the plate was determined by reading the generated fluorescence.

Statistical analysis. All SNPs were examined for Hardy-Weinberg equilibrium (24). Overall survival (OS) and time to progression (TTP) curves were obtained through the Kaplan-Meier method and the log rank test was used to compare the survival distributions. $\mathrm{P}<0.05$ was considered to indicate a statistically significant result. The Cox regression model was used to test the effect of g.148505302 C $>$ T SNP and prognostic factors on OS.

\section{Results}

Patient characteristics and responses. Patient characteristics are summarized in Table I. The median ECOG performance status and median Ca19.9 level at diagnosis were 
Table II. Position and functional characteristics of the investigated SNPs.

\begin{tabular}{|c|c|c|c|}
\hline SNP & Position & Change & Comments \\
\hline g.148525904C>G & Exon 6 & C:Histidine/G:Aspartate & \\
\hline g.148519011C>T & Intron 8 & $\mathrm{C}: \mathrm{E} 2 \mathrm{~F}-1 \mathrm{TFB}$ & TF expressed in CCA (23) \\
\hline g.148517456T >G & Intron 8 & G: Pax-5 and p53 TFB & TF expressed in CCA (24) \\
\hline g.148505302 C>T & Intron 19 & A:PPAR- $\alpha / R X R-\alpha$ TFB & TF expressed in CCA (25) \\
\hline
\end{tabular}

SNP, single nucleotide polymorphism; TFB, transcription factor binding; PPAR- $\alpha$, peroxisome proliferator-activated receptor- $\alpha$; RXR- $\alpha$, retinoid-X-receptor- $\alpha$; TF, transcription factor; CCA, cholangiocarcinoma.

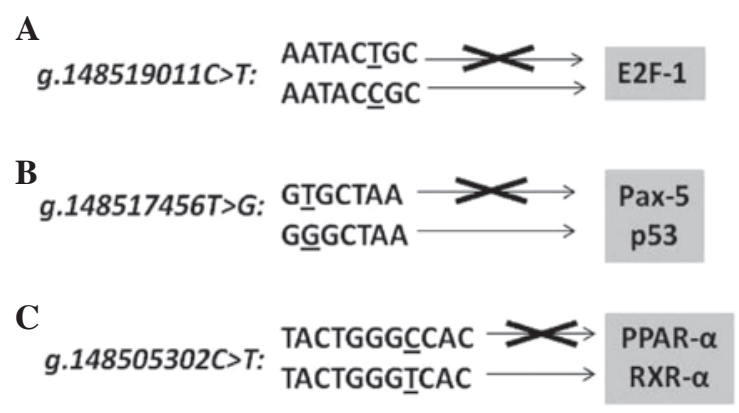

Figure 1. Identification of putative TFBS in DNA sequences. EZH2 SNPs may be responsible for differential TFB. (A) g.148519011 CC allows E2F-1 binding; (B) g.148517456 GG allows Pax-5 and tumor suppressor p53 binding; and (C) g.148505302 TT allows PPAR- $\alpha /$ RXR- $\alpha$ complex binding. TFBS, transcription factor binding sites; EZH2, Enhancer of zeste homolog 2; SNPs, single nucleotide polymorphisms; TFB, transcription factor binding.

1 and $204 \mathrm{IU} / \mathrm{ml}$, respectively. Of the 74 evaluable patients, 3 complete responses (CR) were observed (4.1\%), while a partial response (PR) was observed in 10 of 74 evaluable patients $(13.5 \%)$, stable disease (SD) in 34 patients (45.9\%) and progressive disease (PD) in 27 patients $(36.5 \%)$. A median follow-up of 42.3 months revealed that the median OS was 14.9 months $(8.2-21.6)$ and the 1-year survival rate was $56 \%$.

In silico characterization of g.148505302 $C>T$, g.148525904C>G, g.148519011C>T and $g .148517456 T>G$. Among the 26 SNPs in the EZH2 locus described by Yoon et al (20), only $1 \mathrm{SNP}$ ( $\mathrm{g} .148525904 \mathrm{C}>\mathrm{G}$ ) is located on an exon (exon 6) and is responsible for an amino acid change (histidine/ aspartate). Conversely, 25 SNPs are located in EZH2 non-coding regions and they may affect $\mathrm{EZH} 2$ expression by affecting miRNA, transcription regulator binding or mRNA splicing. However, no SNPs affecting miRNA binding or splicing were identified, while the PROMO 3.0 software detected noteworthy correlations between the allelic variants of 3 of these EZH2 SNPs and TFB sites (Fig. 1). In g.148505302 C $>$ T, the T allele creates a binding site for the peroxisome proliferator-activated receptor (PPAR)- $\alpha$ /retinoid-X-receptor (RXR)- $\alpha$. The $\mathrm{C}$ allele of g.148519011C $>$ T creates a binding site for E2F-1 and the $G$ allele of g.148517456T $>$ G creates a binding site for Pax-5 and p53. All these TFs are expressed in CCA cell lines (25-28).

SNP genotyping. The 4 SNPs selected (Table II) were successfully evaluated in all available DNA samples. The g.148505302 C>T, g.148519011C > T and g.148525904C>G

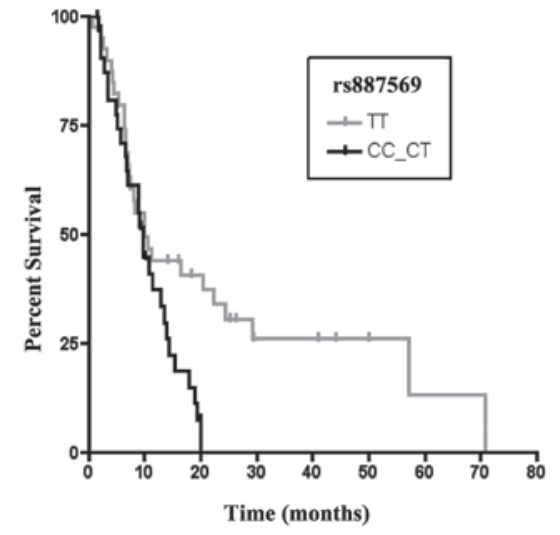

Figure 2. OS according to g.148505302 C >T genotype (rs887569). OS, overall survival.

SNPs were in Hardy-Weinberg equilibrium. Conversely, the g.148517456T>G SNP did not follow the Hardy-Weinberg equilibrium. However, all SNPs had frequencies comparable to those observed in Caucasian populations reported in Pubmed Reference-SNP (RefSNP).

EZH2 SNP correlation with clinical outcome. No significant correlations were identified between the g.148519011C $>\mathrm{T}$, g. $148525904 \mathrm{C}>\mathrm{G}$ and g.148517456T $>\mathrm{G}$ SNPs and clinical outcome, while the g.148505302 C > T (rs887569) SNP had a significant association with OS. In particular, the patients harboring the TT genotype had a significantly longer OS (TT vs. CT-CC, $\mathrm{P}=0.036$; Fig. 2). Moreover, the TT genotype revealed a trend-like correlation with $\mathrm{OS}(\mathrm{P}=0.075)$ in multivariate analysis.

\section{Discussion}

The present study revealed that the g.148505302 $\mathrm{T}$ allele is associated with a longer OS in CCA patients. The g.148505302 C $>$ T SNP is located in intron 19 of the EZH2 gene (20). Through in silico analysis it was revealed that the $\mathrm{T}$ allele creates a binding site for the PPAR- $\alpha / \mathrm{RXR}-\alpha$ heterodimer. The PPARs were identified in 1990 by Issemann and Green (29). PPARs are ligand-activated transcription factors that directly influence the transcription of target genes (30). Three related PPAR isotypes have been identified (PPAR- $\alpha,-\beta / \delta$ and $-\gamma)(31)$. PPAR- $\alpha$ binds to DNA as a heterodimeric complex with RXR- $\alpha$ (32). This complex binds to a specific sequence 
in regulatory regions of target genes, known as peroxisome proliferator response element (PPRE), with two copies of a hexameric nucleotide sequence, TGACCT-like (32). Several studies have suggested differential mechanism underlying the role of PPAR- $\alpha$ in cancer, including key roles in modulation of cell-cycle genes, cell proliferation and cellular apoptosis (33). In accordance with these hypotheses, the g.148505302 T allele, which binds the PPAR- $\alpha /$ RXR- $\alpha$ complex, may trigger mechanisms involved in apoptosis and cell proliferation inhibition by downregulation of the EZH2 oncogene. EZH2 is a transcriptional repressor involved in cell proliferation (19). Overexpression of EZH2 is associated with aggressive and metastatic disease in various types of cancer (34), including CCA. In particular, EZH2 is not expressed in the cholangiocytes or hepatocytes of livers without tumors, but is overexpressed in poorly differentiated carcinoma (35). Therefore, EZH2 expression may be a predictor of the biological aggressiveness and poor prognosis in CCA (36). Cell culture studies have confirmed the expression of EZH2 mRNA in CCA cells, but not in normal cells (35). These studies have also demonstrated that when EZH2 is decreased by suberoylanilide hydroxamic acid (SAHA; a histone deacetylase inhibitor) treatment, the tumor suppressors p16, E-cadherin and p21 are activated (35). Previous studies have shown that EZH2 expression had a stepwise increase in aggressive and invasive CCA (36,37). Since EZH2 drives CSC self-renewal and is associated with poor prognosis in most malignancies, it is conceivable that these cells contribute to the maintenance of the tumoral mass and are implicated in CCA chemoresistance.

EZH2 expression and activity may be affected by functional polymorphisms. SNP genotyping is particularly attractive for tumors detected in the advanced stages, including CCA, since it is an easy-to-perform analysis. This analysis may be performed with a small volume of biological fluids (e.g. $200 \mu \mathrm{l}$ of blood specimens) in less than $24 \mathrm{~h}$.

The current study demonstrates how a candidate EZH2 SNP may be a novel biomarker correlated with clinical outcome in CCA patients. Due to the relatively small sample size and retrospective design, further studies are required in order to validate the prognostic role of this SNP in CCA. Our in silico prediction should also be extended by appropriate molecular analyses, which go beyond the scope of the present analysis. In addition, the possibility that the g.148505302 C>T SNP is in linkage disequilibrium with other polymorphic variants, which may be responsible for the prognostic significance of this marker, cannot be ruled out.

In conclusion, to the best of our knowledge, this is the first study to show an EZH2 SNP having a significant impact on CCA outcome, possibly through its role in the PPAR- $\alpha /$ RXR- $\alpha$ complex interaction with EZH2. If these results are confirmed by larger prospective studies, this EZH2 polymorphism may be useful for predicting the clinical outcome in CCA patients.

\section{Acknowledgements}

This study was partially supported by grants from the Netherlands Organization for Scientific Research (NWO) to Dr Elisa Giovannetti and from the Ministero dell'Istruzione, dell'Universita' e Ricerca (PRIN 2008) to Dr Elisa Giovannetti and Professor Romano Danesi.

\section{References}

1. Parkin DM, Ohshima H, Srivatanakul P and Vatanasapt V: Cholangiocarcinoma: epidemiology, mechanisms of carcinogenesis and prevention. Cancer Epidemiol Biomarkers Prev 2: 537-544, 1993

2. Jemal A, Bray F, Center MM, Ferlay J, Ward E and Forman D: Global cancer statistics. CA Cancer J Clin 61: 69-90, 2011.

3. Welzel TM, Graubard BI, El-Serag HB, Shaib YH, Hsing AW, Davila JA and McGlynn KA: Risk factors for intrahepatic and extrahepatic cholangiocarcinoma in the United States: a population-based case-control study. Clin Gastroenterol Hepatol 5: 1221-1228, 2007.

4. Shaib YH, El-Serag HB, Nooka AK, Thomas M, Brown TD, Patt YZ and Hassan MM: Risk factors for intrahepatic and extrahepatic cholangiocarcinoma: a hospital-based case-control study. Am J Gastroenterol 102: 1016-1021, 2007.

5. Albores-Saavedra J, Murakata L, Krueger JE and Henson DE: Noninvasive and minimally invasive papillary carcinomas of the extrahepatic bile ducts. Cancer 89: 508-515, 2000.

6. Farges $\mathrm{O}$ and Fuks D: Clinical presentation and management of intrahepatic cholangiocarcinoma. Gastroenterol Clin Biol 34: 191-199, 2010.

7. Lim JH and Park CK: Pathology of cholangiocarcinoma. Abdom Imaging 29: 540-547, 2004.

8. Gatto $\mathrm{M}$ and Alvaro D: New insights on cholangiocarcinoma. World J Gastrointest Oncol 2: 136-145, 2010.

9. Leelawat K, Narong S, Wannaprasert J and Ratanashu-ek T: Prospective study of MMP7 serum levels in the diagnosis of cholangiocarcinoma. World J Gastroenterol 16: 4697-4703, 2010.

10. Seehofer D, Kamphues C and Neuhaus P: Management of bile duct tumors. Expert Opin Pharmacother 9: 2843-2856, 2008.

11. de Groen PC, Gores GJ,LaRusso NF, Gunderson LL and Nagorney DM: Biliary tract cancers. N Engl J Med 341: 1368-1378, 1999.

12. Valk-Lingbeek ME, Bruggeman SW and van Lohuizen M: Stem cells and cancer; the polycomb connection. Cell 118: 409-418, 2004.

13. Lund $\mathrm{AH}$ and van Lohuizen M: Polycomb complexes and silencing mechanisms. Curr Opin Cell Biol 16: 239-246, 2004.

14. Mathews LA, Crea F and Farrar WL: Epigenetic gene regulation in stem cells and correlation to cancer. Differentiation 78: 1-17, 2009.

15. Liu DC and Yang ZL: Overexpression of EZH2 and loss of expression of PTEN is associated with invasion, metastasis and poor progression of gallbladder adenocarcinoma. Pathol Res Pract 207: 472-478, 2011.

16. Varambally S, Dhanasekaran SM, Zhou M, Barrette TR, Kumar-Sinha C, Sanda MG, Ghosh D, Pienta KJ, Sewalt RG, Otte AP, Rubin MA and Chinnaiyan AM: The polycomb group protein EZH2 is involved in progression of prostate cancer. Nature 419: 624-629, 2002.

17. Kleer CG, Cao Q, Varambally S, Shen R, Ota I, Tomlins SA, Ghosh D, Sewalt RG, Otte AP, Hayes DF, Sabel MS, Livant D, Weiss SJ, Rubin MA and Chinnaiyan AM: EZH2 is a marker of aggressive breast cancer and promotes neoplastic transformation of breast epithelial cells. Proc Natl Acad Sci USA 100: 11606-11611, 2003.

18. Jacobs JJ, Kieboom K, Marino S, DePinho RA and van Lohuizen M: The oncogene and Polycomb-group gene bmi-1 regulates cell proliferation and senescence through the ink $4 \mathrm{a}$ locus. Nature 397: 164-168, 1999.

19. Sasaki M, Yamaguchi J, Itatsu K, Ikeda H and Nakanuma Y: Over-expression of polycomb group protein EZH2 relates to decreased expression of p16 INK4a in cholangiocarcinogenesis in hepatolithiasis. J Pathol 215: 175-183, 2008.

20. Yoon KA, Gil HJ, Han J, Park J and Lee JS: Genetic polymorphisms in the polycomb group gene EZH2 and the risk of lung cancer. J Thorac Oncol 5: 10-16, 2010.

21. Messeguer X, Escudero R, Farré D, Núñez O, Martinez J and Albà MM: PROMO: detection of known transcription regulatory elements using species-tailored searches. Bioinformatics 18: 333-334, 2002.

22. Farré D, Roset R, Huerta M, Adsuara JE, Rosello L, Albà MM and Messeguer X: Identification of patterns in biological sequences at the ALGGEN server: PROMO and MALGEN. Nucleic Acids Res 31: 3651-3653, 2003.

23. Barenboim M, Zoltick BJ, Guo Y and Weinberger DR: MicroSNiPer: a web tool for prediction of SNP effects on putative microRNA targets. Hum Mutat 31: 1223-1232, 2010. 
24. Rodriguez S, Gaunt TR and Day IN: Hardy-Weinberg equilibrium testing of biological ascertainment for Mendelian randomization studies. Am J Epidemiol 169: 505-514, 2009.

25. Yoon JH, Higuchi H, Werneburg NW, Kaufmann SH and Gores GJ: Bile acids induce cyclooxygenase-2 expression via the epidermal growth factor receptor in a human cholangiocarcinoma cell line. Gastroenterology 122: 985-993, 2002.

26. Kim EM, Kim JS, Choi MH, Hong ST and Bae YM: Effects of excretory/secretory products from Clonorchis sinensis and the carcinogen dimethylnitrosamine on the proliferation and cell cycle modulation of human epithelial HEK293T cells. Korean J Parasitol 46: 127-132, 2008.

27. Zheng T, Wang J, Chen X, Meng X, Song X, Lu Z, Jiang H and Liu L: Disruption of p73-MDM2 binding synergizes with gemcitabine to induce apoptosis in HuCCT1 cholangiocarcinoma cell line with p53 mutation. Tumour Biol 31: 287-295, 2010.

28. Han C, Lim K, Xu L, Li G and Wu T: Regulation of Wnt/ beta-catenin pathway by cPLA2alpha and PPARdelta. J Cell Biochem 105: 534-545, 2008.

29. Issemann I and Green S: Activation of a member of the steroid hormone receptor superfamily by peroxisome proliferators. Nature 347: 645-650, 1990.

30. Evans RM: The steroid and thyroid hormone receptor superfamily. Science 240: 889-895, 1988

31. Youssef $\mathrm{J}$ and Badr M: Enhanced hepatocarcinogenicity due to agonists of peroxisome prolifer-atoractivated receptors in senescent rats: role of peroxisome proliferation, cell proliferation and apoptosis. ScientificWorldJournal 2: 1491-1500, 2002.
32. Kliewer SA, Umesono K, Noonan DJ, Heyman RA and Evans RM: Convergence of 9-cis retinoic acid and peroxisome proliferator signalling pathways through heterodimer formation of their receptors. Nature 358: 771-774, 1992.

33. Tugwood JD, Issemann I, Anderson RG, Bundell KR, McPheat WL and Green S: The mouse peroxisome proliferator activated receptor recognizes a response element in the 5 flanking sequence of the rat acyl CoA oxidase gene. EMBO J 11: 433-439, 1992.

34. Chase A and Cross NC: Aberrations of EZH2 in cancer. Clin Cancer Res 17: 2613-2618, 2011.

35. Yamaguchi J, Sasaki M, Sato Y, Itatsu K, Harada K, Zen Y, Ikeda $H$, Nimura $Y$, Nagino $M$ and Nakanuma $Y$ : Histone deacetylase inhibitor (SAHA) and repression of EZH2 synergistically inhibit proliferation of gallbladder carcinoma. Cancer Sci 101: 355-362, 2010.

36. Sasaki M, Ikeda H, Itatsu K, Yamaguchi J, Sawada S, Minato H, Ohta $\mathrm{T}$ and Nakanuma Y: The overexpression of polycomb group proteins Bmi1 and EZH2 is associated with the progression and aggressive biological behavior of hepatocellular carcinoma. Lab Invest 88: 873-882, 2008.

37. Nakanuma Y, Sasaki M, Sato Y, Ren X, Ikeda H and Harada K: Multistep carcinogenesis of perihilar cholangiocarcinoma arising in the intrahepatic large bile ducts. World J Hepatol 1: 35-42, 2009. 